\title{
Addressing Inpatient Admissions and Readmissions Due to Adverse Drug Reactions in the Oldest Old
}

\author{
Xavier Corbella \\ Internal Medicine Department, Bellvitge University Hospital, Barcelona, Spain; Faculty of Medicine and Health Sciences, \\ Universitat Internacional de Catalunya, Barcelona, Spain.
}

Received: $12 / 08 / 2015$

Accepted: 19/09/2015

Published: 24/09/2015

How to cite this article: Corbella X. Addressing inpatient admissions and readmissions due to adverse drug reactions in the oldest old. EJCRIM 2015;2:DOI:10.12890/2015_S1SP08

Conflicts of Interests: The authors declare that they have no conflicts of interest in this research.

Acknowledgements: This abstract has been presented as a lecture at $2^{\text {nd }}$ International Seminar REPOSI: Targeting the burden of polypharmacy in the elderly.

This article is licensed under a Commons Attribution Non-Commercial 4.0 License

Adverse drugs reactions (ADRs) in the older population are a major healthcare problem resulting in significant morbidity, healthcare consumption and high costs. In accordance, all ADR-related admissions of patients aged $\geq 65$ years are prospectively identified through a systematic daily review of admission diagnosis of all patients urgently hospitalized at the Bellvitge University Hospital, a tertiary care public institution in Barcelona, Spain. Furthermore, we undertook a cross-sectional study through the database of the Pharmacovigilance Program for assessing the prevalence and mortality rates of urgent hospitalization due to ADRs in patients aged $\geq 65$ years. From 2008 to 2014 , ADRs were suspected to be the main reason for urgent admission in 1,976 out of 60,263 patients aged $\geq 65$ years (prevalence of ADRrelated hospitalization: $3.3 \%$ [95\% CI 3.1-3.4\%]). The crude in-hospital mortality rate was $10.2 \%$ in patients with ADR-related admission and $9 \%$ in patients admitted for other causes $(p=0.077)$. Most patients $(86 \%)$ were exposed to polypharmacy and a drug-drug interaction was suspected in $49 \%$ of cases. The most frequent drug-reaction associations were acute renal failure related to renin-angiotensin system (RAS) inhibitors, gastrointestinal bleeding caused by antithrombotics and/or non-steroidal anti-inflammatories, and intracranial bleeding induced by vitamin $\mathrm{K}$ antagonists. 